Proc. XII Int. School on Theoretical Physics - Symmetry and Structural Properties of Condensed Matter

\title{
Magnetic Pentagonal Ring Galois Extensions and Crystallography
}

\author{
M. LABUZ ${ }^{a, *}$ AND T. LULEK ${ }^{b, c}$ \\ ${ }^{a}$ Department of Theoretical Physics, Faculty of Mathematics and Natural Sciences, University of Rzeszów; \\ S. Pigonia 1, 35-310 Rzeszów, Poland \\ ${ }^{b}$ Faculty of Physics, Adam Mickiewicz University, Umultowska 85, 61-614 Poznań, Poland \\ ${ }^{c}$ Department of Experimental Physics, Faculty of Mathematics and Natural Sciences, University of Rzeszów; \\ S. Pigonia 1, 35-310 Rzeszów, Poland
}

\begin{abstract}
The Galois symmetry of exact Bethe Ansatz eigenstates for magnetic pentagonal ring is shown to bear a close analogy to some crystallographic constructions. Automorphisms of number field extensions associated with these eigenstates prove to be related to choices of the Bravais cells in the finite crystal lattice $\mathbb{Z}_{2} \times \mathbb{Z}_{2}$, responsible for extension of the cyclotomic field by the Bethe parameters.
\end{abstract}

DOI: 10.12693/APhysPolA.132.97

PACS/topics: 75.10.Jm, 03.65.Fd, 03.67.Lx, 02.10.Ud, 02.10.De

\section{Introduction}

It is well known that the eigenproblem of the Heisenberg Hamiltonian for the ring of $N$ nodes with the spin- $\frac{1}{2}$ and isotropic nearest neighbour interactions (the XXX model) [1] is expressible in terms of integers, and therefore its exact solution needs a finite extension of the prime field $\mathbb{Q}$ of rationals [2]. The aim of the present note is to point out some consequences of this observation for the case of $N=5$, i.e. for the magnetic pentagonal ring. Eigenstates and eigenvalues for this case were presented in $[3]$ in terms of cyclotomic number field $\mathbb{Q}(\omega)$, $\omega=\exp (2 \pi \mathrm{i} / 5)$, i.e. the extension of $\mathbb{Q}$ by the primitive fifth root of unity, associated with the Fourier transform for this case. $\mathbb{Q}(\omega)$ can be seen as the complex Heisenberg number field, that is the minimal field sufficient to express the solution of the eigenproblem of the Heisenberg Hamiltonian. Still, $\mathbb{Q}(\omega)$ is not sufficient to express this solution in the form prescribed by the Bethe Ansatz [4], that is in terms of spectral parameters of the Bethe pseudoparticles, or, equivalently, of the corresponding pseudomomenta or portions of phase. It was shown in [3] that the standard Bethe Ansatz presentation requires a further extension of the complex Heisenberg field to the so-called Bethe number field $\mathbb{B}$, within the procedure referred there to as the inverse Bethe Ansatz: how to determine the Bethe parameters (pseudomomenta, etc.) once the exact solution of the Heisenberg eigenproblem is already known. The inverse Bethe Ansatz for $N=5$ was solved in [3], and the Galois symmetry associated with this case was thoroughly discussed in [5], in terms of a short exact sequence of automorphism group of consecutive field extensions, and appropriate homomorphism

\footnotetext{
*corresponding author; e-mail: labuz@ur.edu.pl
}

groups. This short exact sequence bears a strong analogy with crystallographic constructions of space groups as extensions of translation groups by point groups.

In the present note we discuss in some detail this analogy of number field extensions to crystallography. We focus on discussion of the corresponding arithmetic classes of all possible actions of the Galois group of the cyclotomic field on the quotient $\mathbb{B} / \mathbb{Q}(\omega)$, associated with choices of a Bravais cell within the two-dimensional lattice with the Born-Karman period two. The group of all automorphisms of such a lattice is $G L\left(2, \mathbb{Z}_{2}\right)$, and the action which defines the Galois group of the Bethe field $\mathbb{B}$ arises as one of three possible embeddings. We present a transparent construction of these embeddings.

\section{Galois extensions and crystallography}

The analogy between Galois extensions associated with Bethe Ansatz eigenstates and crystallography bases on comparison of two exact sequences of groups and homomorphisms (we use here the notation of [5])

$$
\begin{aligned}
& \quad 1_{\text {mult }} \longrightarrow D_{2} \stackrel{\kappa}{\longrightarrow} G \stackrel{\omega}{\longrightarrow} C_{4} \longrightarrow 1_{\text {mult }} \\
& \text { and } \\
& \quad 0_{\text {add }} \longrightarrow T \stackrel{\kappa}{\longrightarrow} G \stackrel{\omega}{\longrightarrow} Q \longrightarrow 1_{\text {mult }},
\end{aligned}
$$

where $D_{2}=\operatorname{Aut}(\mathbb{B} / \mathbb{Q}(\omega))$ and $C_{4}=\operatorname{Aut}(\mathbb{Q}(\omega) / \mathbb{Q})$ are the Galois groups of appropriate field extensions, written in the Schoenflies notation as the corresponding point groups, whereas $T$ and $Q$ are the corresponding translation and point group, respectively, of a crystal lattice. Moreover, $1_{\text {mult }}$ and $0_{\text {add }}$ is the trivial (one-element) group in the multiplicative and additive notation, respectively. Arrows in Eqs. (1) and (2) denote group homomorphisms, such that the image of each left morphisms coincides with the kernel of its right neighbour. Thus, in particular, $\kappa: T \rightarrow G$ is the monomorphism (injection), whereas $\omega: G \rightarrow Q$ is the epimorphism (surjection) (cf., 
e.g. Lang [6] and Mozrzymas [7, 8] for algebraic and crystallographic definitions).

We describe shortly the arithmetic meaning of the active group $C_{4}$ and passive $D_{2}$ of the exact sequence (1), arising from the Heisenberg eigenproblem of pentagon. The cyclotomic field $\mathbb{Q}(\omega)$ is a linear space over $\mathbb{Q}$, of dimension 4 , with the cyclic basis $\left(1, \omega, \omega^{2}, \omega^{3}\right)$, and thus the active group $C_{4}=\left\{\tau_{l} \mid l= \pm 1, \pm 2\right\}$ is generated by the automorphism $\tau_{2}$ which sends the radical $\omega$ to $\omega^{2}$. It is given by the matrix

$$
\tau_{2}=\left(\begin{array}{llll}
1 & 0 & -1 & 0 \\
0 & 0 & -1 & 1 \\
0 & 1 & -1 & 0 \\
0 & 0 & -1 & 0
\end{array}\right)
$$

in the cyclic basis. We also recall here that the active group $C_{4}$ acts regularly on the interior $\{k= \pm 1, \pm 2\}$ of the Brillouin zone $B=\{0, \pm 1, \pm 2\}$ of the pentagon, such that $\tau_{1}$ is the identity, $\tau_{-1}$ reverses the sign of the quasimomentum $k \rightarrow-k$, and the squares $\tau_{2}$ and $\tau_{-2}$, change also the values of quasimomenta $(k \rightarrow \pm 2 k \bmod 5)$.

The passive group $D_{2}$ arises from the inverse Bethe Ansatz for the case of two spin deviations in pentagon, which results in a quadratic equation

$$
\left(1+\omega^{k}\right) a^{2}-(E+4) a+\left(1-\omega^{k}\right)=0
$$

for the portion of phase

$$
a=\mathrm{e}^{\mathrm{i} p}=\frac{\lambda+\mathrm{i} / 2}{\lambda-\mathrm{i} / 2}
$$

of a Bethe pseudoparticle in the state with given energy $E$ and quasimomentum $k$ (recall that such a classification of exact eigenstates is unique in the two-magnon sector of pentagon, cf. Table I).

TABLE I

Classification of BA eigenstates for pentagon for the highest weight vectors $r^{\prime}=r=2 . \nu \vdash r^{\prime}$ denotes the Young diagram of a partition of $r^{\prime}$, and $\nu \mathcal{L}$ is a rigged string configuration equipped with total quasimomentum $k$.

\begin{tabular}{lrr}
\hline \hline$\nu \vdash r^{\prime}$ & \multicolumn{1}{l}{$\nu \mathcal{L}$} & $k$ \\
\hline$\{2\}$ & 2 & 2 \\
$\left\{1^{2}\right\}$ & -2 & 1 \\
& -2 & \\
$\left\{1^{2}\right\}$ & -2 & 0 \\
& 2 & \\
$\left\{1^{2}\right\}$ & 2 & -1 \\
$\{2\}$ & 2 & -2 \\
& -2 &
\end{tabular}

In Eq. (5), $p$ denotes the pseudomomentum, and $\lambda$ the spectral parameter of a Bethe pseudoparticle. Each row in Table I contains a state of the highest weight for the pentagonal magnet, parametrized by rigged string configurations $\nu \mathcal{L}$, with $\nu$ being the partition of $r^{\prime}=r=$ 2 and denoting the string configuration, while $\mathcal{L}$ describes the unique collection of riggings $[9,10]$. It follows that all roots of $\mathrm{Eq}$ (4) for the interior $\{k= \pm 1, \pm 2\}$ of the Brillouin zone of pentagon span the number field $\mathbb{B} / \mathbb{Q}(\omega)$, which is a linear space over the cyclotomic field $\mathbb{Q}(\omega)$, of dimension four, with the basis $\left(1, \gamma_{1}, \gamma_{-1}, \gamma_{1} \gamma_{-1}\right)$, where

$$
\gamma_{1}=\sqrt{-1-2 \sqrt{5}}, \quad \gamma_{-1}=\sqrt{-1+2 \sqrt{5}}
$$

are radicals associated with Eq. (4) for $k= \pm 1$ (squares in $C_{4}$ ) and $k= \pm 2$ (non-squares in $C_{4}$ ), respectively.

The above basis of the quotient number field $\mathbb{B} / \mathbb{Q}(\omega)$ can be intepreted, after change to additive notation as a two-dimensional module $\mathbb{Z}_{2} \times \mathbb{Z}_{2}$ over the cyclotomic field (observe that $\gamma_{1}^{2} \in \mathbb{Q}(\omega), \gamma_{-1}^{2} \in \mathbb{Q}(\omega)$ ), generated by any ordered pair $\left(a_{1}, a_{2}\right)$ from the set $\left\{\gamma_{1}, \gamma_{-1}, \gamma_{1} \gamma_{-1}\right\}$. In other words, this basis constitutes a two-dimensional crystal lattice with the Born-Karman period 2 (cf. Fig. 1), and ordered pair $\left(a_{1}, a_{2}\right)$ defines a Bravais cell for $\mathbb{Z}_{2} \times \mathbb{Z}_{2}$ (cf. Fig. 2). Thus

$$
\text { Aut } D_{2}=\left\{\begin{array}{l}
\tau_{x y}=\left(\begin{array}{ll}
1 & 0 \\
0 & 1
\end{array}\right) ; \tau_{y x}=\left(\begin{array}{ll}
0 & 1 \\
1 & 0
\end{array}\right) ; \\
\tau_{x z}=\left(\begin{array}{ll}
1 & 1 \\
0 & 1
\end{array}\right) ; \tau_{z x}=\left(\begin{array}{ll}
1 & 1 \\
1 & 0
\end{array}\right) ; \\
\tau_{y z}=\left(\begin{array}{ll}
0 & 1 \\
1 & 1
\end{array}\right) ; \tau_{z y}=\left(\begin{array}{ll}
1 & 0 \\
1 & 1
\end{array}\right) .
\end{array}\right\},
$$

is the group of all automorphisms of the lattice $\mathbb{Z}_{2} \times \mathbb{Z}_{2}$. It is well known that the crystallographic space groups in $n$ dimensions are defined in terms of actions of the active group $Q$ on the lattice $\mathbb{Z}^{n}$, i.e. homomorphisms $\Delta: Q \rightarrow$ Aut $\mathbb{Z}^{n} \equiv G L(n, \mathbb{Z})$.

$\begin{array}{cc}U_{y} \bullet \gamma_{-1} & U_{z} \bullet \gamma_{1} \gamma_{-1} \\ 0,1 & 1,1 \\ & \\ E \bullet 1 & U_{x} \bullet \gamma_{1} \\ 0,0 & 1,0\end{array}$

Fig. 1. The periodic square $\mathbb{Z}_{2} \times \mathbb{Z}_{2}$ as a regular orbit of the passive group $D_{2}$. Elements of the group Aut $D_{2}=G L\left(2, \mathbb{Z}_{2}\right)$ are classified by pairs from the set $\left\{U_{x}, U_{y}, U_{z}\right\}$, corresponding to the choice of a Bravais cell of $\mathbb{Z}_{2} \times \mathbb{Z}_{2}$ as a two-dimensional lattice with the BornKarman period $N=2$.

It is clear that the analogs for the pentagonal ring are homomorphisms $\Delta: C_{4} \rightarrow G L\left(2, \mathbb{Z}_{2}\right)$. Each such action is readily defined by prescribing its value on the generator $\tau_{2} \in C_{4}$ of the active group $C_{4}$. The simplest possibility is the trivial action, namely

$$
\Delta_{1}\left(\tau_{2}\right)=\tau_{x y}=\left(\begin{array}{ll}
1 & 0 \\
0 & 1
\end{array}\right),
$$

which yields $G_{1}=C_{4} \times D_{2}$, the direct product of the active and the passive group. The other three possibilities 


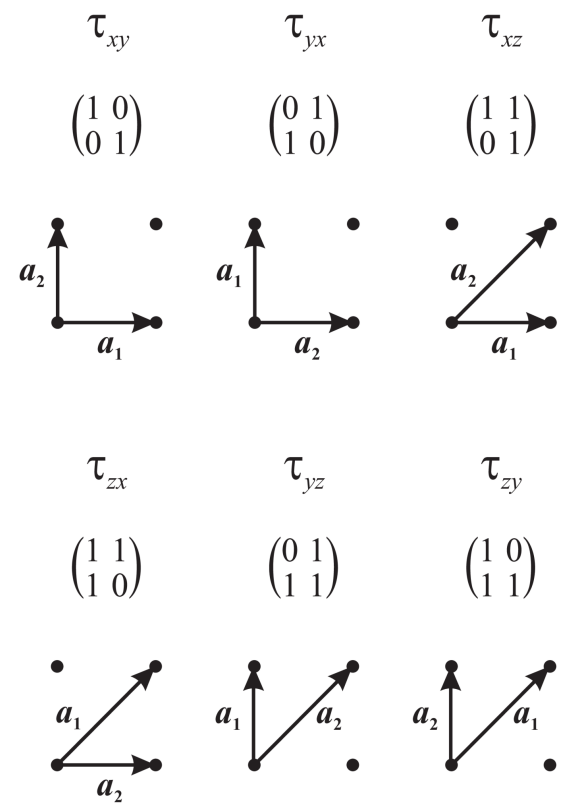

Fig. 2. Six possible choices of a basis on the lattice $\mathbb{Z}_{2} \times \mathbb{Z}_{2}$.

$$
\begin{aligned}
& \Delta_{2}\left(\tau_{2}\right)=\tau_{y x}=\left(\begin{array}{ll}
0 & 1 \\
1 & 0
\end{array}\right) ; \\
& \Delta_{3}\left(\tau_{2}\right)=\tau_{y x}=\left(\begin{array}{ll}
1 & 1 \\
1 & 0
\end{array}\right) ; \\
& \Delta_{4}\left(\tau_{2}\right)=\tau_{y x}=\left(\begin{array}{ll}
1 & 0 \\
1 & 1
\end{array}\right),
\end{aligned}
$$

are mutually conjugated by inner automorphisms of the group $G L\left(2, \mathbb{Z}_{2}\right)$, and yield a non-trivial extension, namely the Galois group $G$ of the Bethe number field,

$$
G=\operatorname{Aut}(\mathbb{B} / \mathbb{Q}(\omega)), \quad|G|=16,
$$

described in detail in [5]. Each time, it is a semidirect product of the active and the passive group, with appropriate action. All these semidirect products are mutually isomorphic. It is worth to add that the analogy of the Galois group $G$ of the Bethe field $\mathbb{B}$ with space groups results to be somehow trivial, since $G$ does not admit any non-trivial factor system.

\section{Conclusions}

We conclude that the pair $\left(C_{4}, D_{2}\right)$ yields two arithmetic classes of group extensions, given by Eqs. (8) and (9). The first class $\Delta_{1}$ gives the direct product, which is not consistent with the Galois symmetry of the Bethe number field $\mathbb{B}$, whereas the second class $\left\{\Delta_{2}, \Delta_{3}, \Delta_{4}\right\}$, yields three isomorphic groups, and the first of them, of which, $\Delta_{2}$, realizes the Galois automorphisms of $\mathbb{B}$, consistent with our choice of radicals $\omega, \gamma_{1}, \gamma_{-1}$ generating $\mathbb{B}$. We hope that the analogy between the Galois symmetry of exact Bethe Ansatz eigenstates and crystallographic constructions displayed here might shed some light on structural properties to both items. We also wish to observe that the Born-Karman periodic boundary conditions, which seem to be somehow artificial in the midst of crystallography and physics of bulk solids, arise in a natural way within finite extensions of the prime field $\mathbb{Q}$ of rationals in integrable systems.

\section{References}

[1] W. Hao, R.I. Nepomechie, A.J. Sommese, Phys. Rev. E 88, 052113 (2013)..

[2] J. Milewski, G. Banaszak, T. Lulek, M. Łabuz, R. Stagraczyński, OSID 19, 1250012 (2012)..

[3] J. Milewski, G. Banaszak, T. Lulek, M. Labuz, Physica B 406, 520 (2011)..

[4] H. Bethe, Z. Phys. 71, 205 (1931) (in German; English translation in: D.C. Mattis, The Many-Body Problem, World Sci., Singapore 1993, p. 689.).

[5] G. Banaszak, B. Lulek, T. Lulek, J. Milewski, B. Szydło, ROMP 71, 205 (2013)..

[6] S. Lang, Algebra, Addison-Wesley World Student Series, Reading (MA) 1970.

[7] J. Mozrzymas, Applications of Group Theory in Physics, PWN, Warsaw 1977 (in Polish).

[8] J. Mozrzymas, ROMP 6, 29 (1974); ROMP 6, 83 (1974).

[9] B. Lulek, T. Lulek, M. Łabuz, R. Stagraczyński, Physica B 405, 2654 (2010)..

[10] P. Jakubczyk, A. Wal, D. Jakubczyk, T. Lulek, Comp. Phys. Commun. 183, 1354 (2012).. 